\title{
Nucleosome-mediated synergism between transcription factors on the mouse mammary tumor virus promoter
}

\author{
(chromatin structure/glucocorticoid receptor/histone depletion/nuclear factor 1/genomic footprinting)
}

\author{
Sebastián Chávez* and Miguel Beato ${ }^{\dagger}$ \\ Institut für Molekularbiologie und Tumorforschung, E.-Mannkopff-Strasse, 2, D-35033 Marburg, Germany \\ Communicated by Pierre Chambon, College de France, Strasbourg, France, January 10, 1997 (received for review September 6, 1996)
}

\begin{abstract}
In unstimulated mammalian cells and in Saccharomyces cerevisiae, the mouse mammary tumor virus (MMTV) promoter is silent and organized into positioned nucleosomes, one of which encompasses the binding sites for glucocorticoid receptor (GR) and nuclear factor I (NFI). Glucocorticoid induction in vivo involves a functional synergism between GR and NFI and simultaneous occupancy of the promoter sites for both proteins that cannot be reproduced on naked DNA. The role of chromatin in the process of induction was investigated by manipulating the nucleosome density in yeast strains carrying a regulated histone $\mathrm{H} 4$ gene. Following depletion of nucleosomes, independent transactivation by NFI or by GR, as well as binding of the individual proteins to the MMTV promoter, were enhanced, in agreement with a repressive function of nucleosomes. In contrast, NFI-dependent hormone induction of the promoter and the simultaneous binding of receptor and NFI were compromised by nucleosome depletion. This effect could be partly mediated by a cryptic binding site for the receptor that is functional only in the nucleosomal context. Thus, positioned nucleosomes do not only account for constitutive repression of the MMTV promoter, but also participate in induction by mediating cooperative binding and functional synergism between GR and NFI.
\end{abstract}

The organization of eukaryotic DNA in chromatin is usually seen as an obstacle for binding of transcription factors to their cognate sequences and is supposed to participate in constitutive repression of genes as well as in gene induction by derepession $(1,2)$. However, nucleosomes can also fulfill a positive architectural function by organizing the DNA sequences so as to permit interactions between factors bound to otherwise distant sites (3-5). A rapidly growing number of reports document the participation of chromatin dynamics in the process of gene regulation (for a recent review, see ref. 6), and direct interactions between transcription factors and chromatin components have been described $(7,8)$. Although in most cases these interactions are involved in transcriptional repression, a positive effect in particular contexts cannot be excluded (9)

To analyze the participation of nucleosomes in gene expression, we have chosen the mouse mammary tumor virus (MMTV) promoter which is regulated by steroid hormones through an interaction of the hormone receptors with a complex array of hormone responsive elements (HREs) located between -190 and -80 (see ref. 10 and references therein). Hormonal induction of the MMTV promoter requires not only binding of the hormone receptors to the HREs but also of other transcription factors to adjacent promoter

The publication costs of this article were defrayed in part by page charge payment. This article must therefore be hereby marked "advertisement" in accordance with 18 U.S.C. $\$ 1734$ solely to indicate this fact.

Copyright (C) 1997 by The National Academy of Sciences of the USA 0027-8424/97/942885-6\$2.00/0

PNAS is available online at http://www.pnas.org sequences (11). In particular, a functional synergism with members of the ubiquitous nuclear factor I (NFI) family bound to a site immediately downstream of the HREs is essential for strong induction in vivo $(12,13)$. Conventional mechanisms invoking cooperative DNA binding are insufficient to explain this synergism between glucocorticoid receptor (GR) and NFI, since both proteins compete for binding to naked promoter DNA (13). In cell-free transcription assays with naked DNA templates, NFI acts as a strong constitutive transcription factor but does not synergize with hormone receptors $(11,14)$. Thus, the mechanism underlying the functional synergism between GR and NFI during hormonal induction remains obscure.

In mice fibroblasts (15), in unstimulated breast epithelial cells (16), as well as when introduced into Saccharomyces cerevisiae (17), the MMTV promoter is silent and organized into positioned nucleosomes. Although the precise positioning is still controversial (18), we find a dominant frame that encompasses the binding sites for GR and NFI in yeast and in mammalian cells $(16,17)$. A similar nucleosome organization is found in chromatin reconstitution experiments with MMTV promoter fragments and core histone octamers (19-22). While GR can access the most distal and the most proximal receptor binding sites, NFI cannot bind to the MMTV promoter incorporated in positioned nucleosomes (19-21), which could be the basis for repression of the promoter in the absence of hormone $(17,23)$. In hormone-induced cells the nucleosome over the promoter is remodeled and all binding sites for receptors as well as the NFI site are occupied, suggesting that mechanisms exist in vivo to permit simultaneous binding of factors to the surface of a nucleosome (16). On the other hand, attempts to demonstrate cooperative binding of GR and NFI to reconstituted nucleosomes have been unsuccessful, and the function of the nucleosome remains unclear (refs. 19 nnd 20; and unpublished results).

To provide genetic evidence for a possible role of nucleosomes in transcriptional control of the MMTV promoter, we have manipulated the nucleosome density in $S$. cerevisiae by modulating transcription of the histone $\mathrm{H} 4$ gene (24). If nucleosomes participate in basal and induced MMTV transcription by determining access of transcription factors, changing the density of nucleosomes on the promoter should influence its activity in the absence and in the presence of hormone. Here we show that nucleosome depletion of yeast strains carrying the MMTV promoter leads to a better accessibility of the promoter for the individual factors, accompanied by a more efficient transactivation by either NFI, NFI-herpes simplex virus activator protein VP16 (VP16), or GR, when

Abbreviations: MMTV, mouse mammary tumor virus; GR, glucocorticoid receptor; NFI, nuclear factor I; HRE, hormone responsive element; VP16, herpes simplex virus activator protein VP16; DMS, dimethyl sulfate; DAC, deacylcortivazol.

*Present address: Departamento de Genética, Facultad de Biología, Universidad de Sevilla, Reina Mercedes, Apartado 1095, E-41080 Seville, Spain. e-mail: schavez@cica.es.

†To whom reprint requests should be addressed. e-mail: beato@imt.uni-marburg.de. 
these factors are expressed individually. These findings support a repressive role of nucleosomes in MMTV promoter function. However, the functional synergism between NFI and GR required for strong hormonal induction, is compromised under conditions of nucleosome depletion, as expected if nucleosomes participate actively in the induction process by facilitating the cooperation between GR and NFI. Possible biochemical mechanisms underlying this positive role of nucleosomes are discussed, taking into consideration a new HRE, which is inactive in transient transfections but contributes to induction in a nucleosomal context.

\section{MATERIALS AND METHODS}

Yeast Strains and Plasmids. Strains SChY50 (MATa, ade2, his3, leu2, lys2, trp1, ura3, thr ${ }^{-}$, tyr ${ }^{-}$, hhf1::HIS3, hhf 2::leu $2 \Delta: A D E 2 /$ pUK421 (CEN TRP1 GAL-HHF2) was generated by disrupting the wild-type LEU2 allele present in strain PKY899 (25) with a leu2 $\Delta: A D E 2$ construct, and replacing plasmid pUK499 by pUK421 (26) following a plasmid shuffle technique. Strain SChY50 is therefore isogenic to UKY403 (26) except that it is $\mathrm{Leu}^{-}$and $\mathrm{Ade}^{+}$, and it carries a single histone $\mathrm{H} 4$ gene that is controlled by the GAL1 promoter. Strain SChY51 was constructed similarly to SChY50 but contains the H4-2 gene controlled by its own promoter.

pLGZ-MMTV (17) was the MMTV-lacZ reporter plasmid used in this work. pSCh105 (23) is a truncated version of the reporter, lacking the HRE region of the MMTV promoter, and pSCh136 is homologous to pLGZ-MMTV, but containing several mutations in the HRE5. pSX26.1 is an HRE-CYC1lac $Z$ reporter (27).

To express NFI we used pAA-CTF2 (28), a vector for the NFI variant CTF2, derived from pAAH5. Plasmid pSCh124 was constructed by inserting a DNA fragment coding for a NFI-VP16 chimera (23) in the expression vector p425MET25 (29). pSCh129 (17) is an expression vector for rat GR.

The HRE5 mutation was constructed by exchanging five positions known to be protected by GR in dimethyl sulfate (DMS) footprinting experiments (30). The wild-type MMTV promoter sequence between -160 and -140 (protected positions shown in boldface type) was changed from GATGTGAGATAAGTGGTTTCC to GATGTAAAACAAGTAATTTCC. This mutations eliminated binding of GR to this site in vitro (data not shown).

Growth and Nucleosome-Depletion Conditions. Yeast strains transformed with the appropriate set of plasmids were grown at $30^{\circ} \mathrm{C}$ in selective medium containing galactose as carbon source. Before reaching stationary phase, cells were washed, transferred to rich medium (yeast extract/ peptone) containing glucose or galactose, and incubated further at $30^{\circ} \mathrm{C}$ for $6 \mathrm{~h}$. For hormone-induction, $0.1 \mu \mathrm{M}$ deacylcortivazol (DAC) or ethanol for control samples were included during this last incubation. After cooling on ice, cells were used for plasmid-superhelicity analysis, micrococcal nuclease digestion, or assayed for $\beta$-galactosidase activity (31).

Genomic Footprinting. For genomic footprinting, strain SChY50, transformed with the appropriate plasmids, was grown at $30^{\circ} \mathrm{C}$ in selective medium containing galactose as carbon source. Before reaching stationary phase, cells were washed and transferred to rich medium containing glucose or galactose. After $1 \mathrm{~h}$ of further incubation at $30^{\circ} \mathrm{C}$, cultures were treated with $1 \mu \mathrm{M}$ DAC or with ethanol for $30 \mathrm{~min}$. Cells were then rapidly harvested, treated with DMS, and processed as described (17). Piperidine-digested DNA samples were then analyzed by linear PCR (17).

\section{RESULTS}

Nucleosome Depletion Facilitates Access to the NFI Site. To study the influence of low nucleosome density on the activity of the MMTV promoter we made use of a yeast strain in which the endogenous histone $\mathrm{H} 4$ genes have been deleted and a GAL1driven histone $\mathrm{H} 4$ gene has been introduced (24). Into this yeast strain we incorporated a MMTV-driven lacZ gene (Fig. 1A). Incubation in glucose medium reduced the nucleosomal density of episomal DNA in this yeast strain, as demonstrated by topoisomer analysis of plasmid DNA (Fig. 1B). Approximately $40 \%$ of the negative superhelical density found in the presence of galactose is lost during nucleosome depletion in the presence of glucose. At the same time, the MMTV promoter sequences became more sensitive to digestion by micrococcal nuclease, as expected for a region partially depleted of nucleosomes (Fig. 1C). This effect is not due to the change in carbon source, which did not influence strains with wild-type $\mathrm{H} 4$ genes.

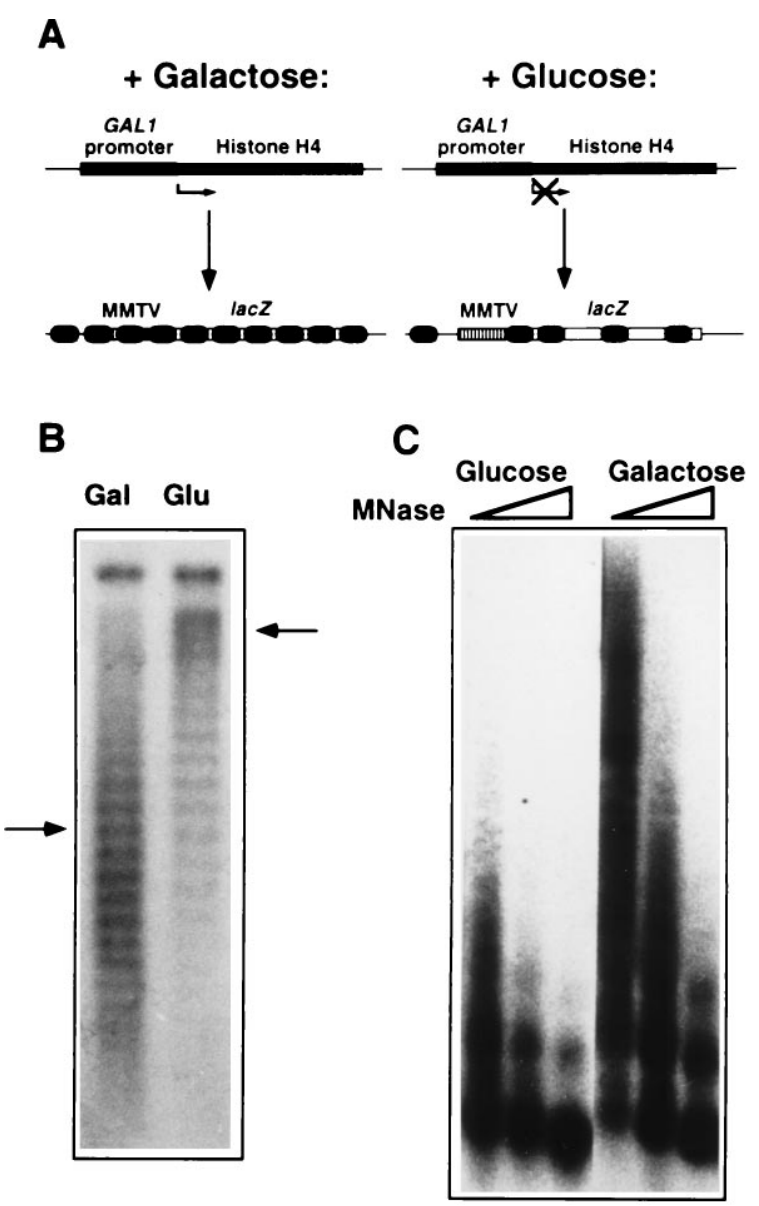

FIG. 1. Repression of histone $\mathrm{H} 4$ gene results in nucleosome depletion over the MMTV promoter. $(A)$ Schematic representation of histone H4-mediated nucleosome depletion, according to the method developed by Grunstein and collaborators (26). (B) Superhelicity of episomes carrying the MMTV-lacZ reporter in SChY50 cells grown in galactose- or glucose-containing media (17). The modal value of the distribution of topoisomers is indicated by arrows. The difference between cells grown in galactose $(\mathrm{Gal})$ and in glucose (Glu) corresponds to $40 \%$ nucleosome depletion. Superhelicity analysis was performed as described (32) but hybridizing with an Escherichia coli lacZ probe. $(C)$ Micrococcal nuclease (MNase) sensitivity of MMTV promoter in normal (galactose) or histone H4-depleted chromatin (glucose) of SChY50 cells (17). Note that in glucose medium the nuclease digestion is more effective and leads to appearance of shorter polynucleosomes and mononucleosomes. MMTV sensitivity to micrococcal nuclease was assayed as described (17) with a probe extending from -173 to -78 in the MMTV promoter. 
A

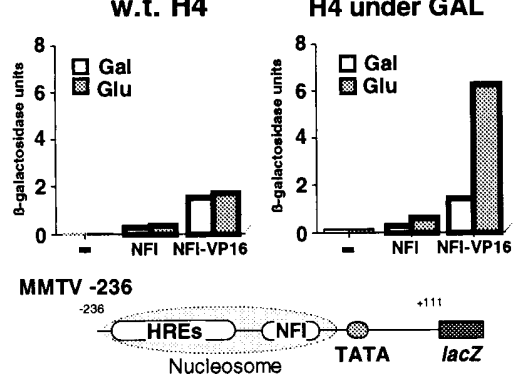

B

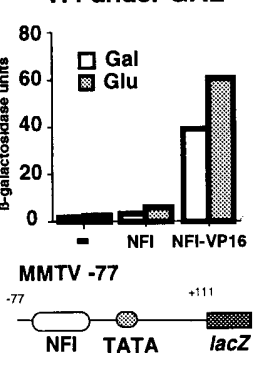

FIG. 2. Nucleosome depletion derepresses NFI-dependent transcription of MMTV promoter. $(A)$ Yeast strains with the histone $\mathrm{H} 4$ gene under its own promoter or under galactose $(\mathrm{Gal})$ control, and containing a complete MMTV-lacZ reporter with a positioned nucleosome, were transformed with an expression vector for NFI, NFI-VP16, or empty vector (23). $\beta$-Galactosidase activities of cultures switched from galactose to glucose (Glu) or kept in galactose are shown. (B) A similar yeast strain with the histone $\mathrm{H} 4$ gene under galactose control and carrying a truncated MMTV-lacZ reporter, depicted at the bottom, was assayed as in $A$. Note the difference in scale between $A$ and $B$.

We have previously shown that in yeast, like in metazoan cells, the MMTV promoter positions a nucleosome which encompasses the HREs and the NFI binding site (17). Under conditions of nucleosome depletion the NFI binding site on the MMTV promoter was more accessible to NFI or a NFI-VP16 chimera, as demonstrated by the enhanced activity of the reporter. The effect observed with NFI was weak because this particular transactivator acts poorly in yeast (28), but it was specific for the nucleosome-depleted strain, as incubation in glucose medium of yeast strains expressing a nonregulated histone $\mathrm{H} 4$ gene had no effect on reporter activity (Fig. 2A). When the more potent chimeric transactivator NFI-VP16 was
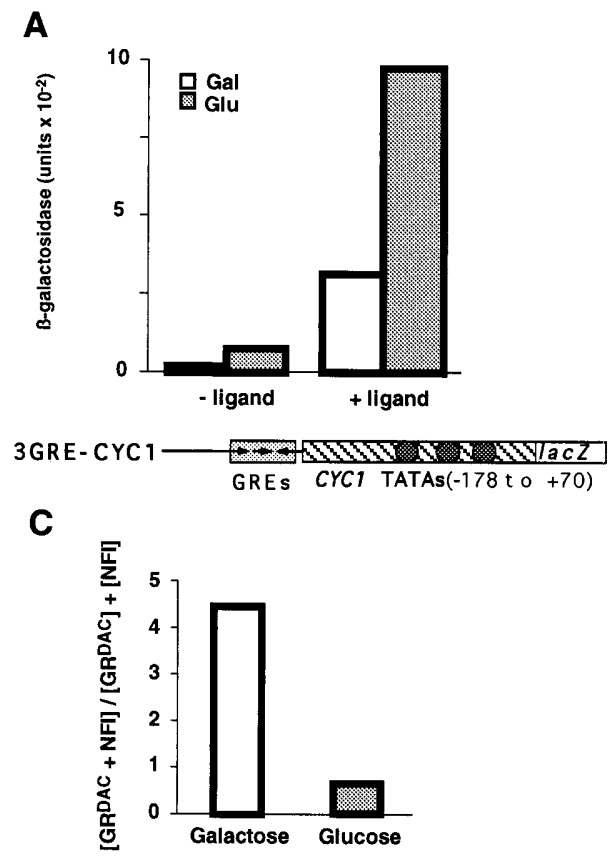

used instead, the effect of nucleosome depletion was more evident and could be quantitated reproducibly. The MMTV reporter was activated 4-fold more efficiently by NFI-VP16 when the nucleosome density was reduced by treatment with glucose. Moreover, genomic footprinting experiments showed partial occupancy of the NFI site on the promoter in the absence of hormone, only under conditions of nucleosome depletion but not at normal nucleosome density (see below and Fig. 4B). Therefore, the physiological nucleosome density contributes to maintaining the NFI site on the MMTV promoter unaccessible for factor binding.

The HRE Region Is Required for Restricting Access to the NFI Site. To ascertain whether access to the NFI site is determined by the sequences encompassing the HREs, we analyzed the response to nucleosome depletion of a truncated MMTV promoter lacking the sequences between -236 and -77 , but containing an intact NFI binding site and core promoter elements. This reporter is already more active in the presence of NFI or NFI-VP16 at normal nucleosome density (23), and its activity is not markedly influenced by nucleosome depletion (Fig. $2 B$; note that the ordinate scale is one order of magnitude higher than in Fig. $2 A$ ). These findings exclude effects of nucleosome depletion due to changes in the levels or the activity of NFI. The levels of NFI mRNA measured by Northern blot analysis were not altered by glucose treatment of yeast strains carrying a regulated histone $\mathrm{H} 4$ gene (data not shown). In contrast with the wild-type promoter (17), indirect end-labeling experiments did not reveal positioning of nucleosomes over the residual MMTV promoter in this truncated construction (data not shown and ref. 23). We conclude that nucleosomes positioned by the region containing the HREs (23) are at least partly responsible for constitutive repression of the MMTV promoter, and that transactivation by NFI is enhanced by nucleosome depletion.

Nucleosome Depletion Improves Transactivation by GR of HRE-Containing Reporters. To test whether nucleosome de-

\section{B}

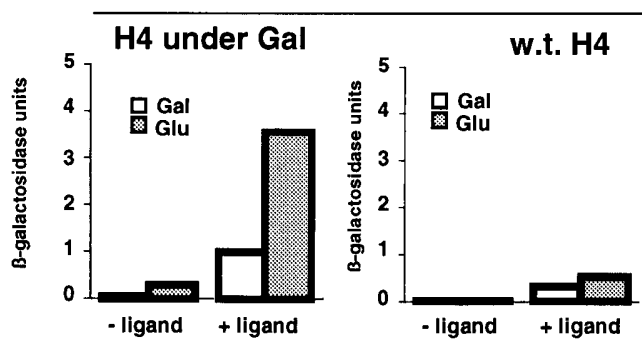

D

GR + NFI

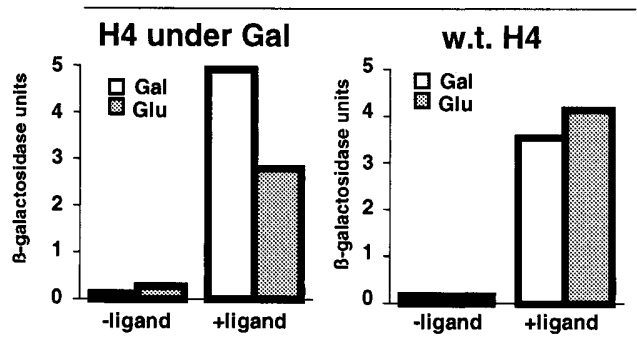

FIG. 3. Nucleosome depletion does not inhibit GR function but hinders the synergistic activation of MMTV promoter by GR and NFI. $(A)$ A yeast strain with the histone $\mathrm{H} 4$ gene under Gal control was transformed with the episomal GRE-lacZ reporter described in the scheme, together with an expression vector for rat GR (27). Cultures were incubated with ligand or vehicle after switching cells from galactose (Gal) to glucose (Glu) or keeping them in galactose. $\beta$-Galactosidase activities are shown. (B Left) $\beta$-Galactosidase activities obtained in experiments similar to those described in $A$ but using the MMTV-lacZ reporter depicted in Fig. 2A. (Right) Results obtained from a yeast strain with the histone $\mathrm{H} 4$ gene driven by its own promoter. $(C)$ Functional synergism between GR and NFI. Synergism is defined as the ratio of the activity found in the presence of GR/DAC and NFI over the sum of the activity found with GR/DAC in the absence of NFI and the activity measured with NFI in the absence of GR/DAC (Fig. 2A Right). (D) Similar experiments to those described in $B$ but in strains additionally transformed with an NFI-expressing vector. 
pletion had an effect on GR function we used a reporter containing several HREs in front of the yeast CYC1 core promoter (27) (Fig. 3A). Constitutive activity as well as GR-mediated induction of this hybrid promoter by the agonistic ligand DAC were enhanced several fold by nucleosome depletion (Fig. $3 A$ ). These results demonstrate that GR can act more efficiently at low nucleosome density on an array of HREs linked to a yeast promoter.

To confirm that this conclusion applies also to the MMTV promoter, we analyzed the effect GR and DAC on MMTV transcription in the absence of NFI. Under these conditions, the observed induction was low, as shown previously (17). As with the CYC1 promoter, transactivation by ligand activated GR is enhanced several fold under conditions of nucleosome depletion (Fig. 3B). Nucleosome depletion did not influence the levels of GR mRNA (data not shown), and glucose treatment of yeast strains expressing the wild-type histone $\mathrm{H} 4$

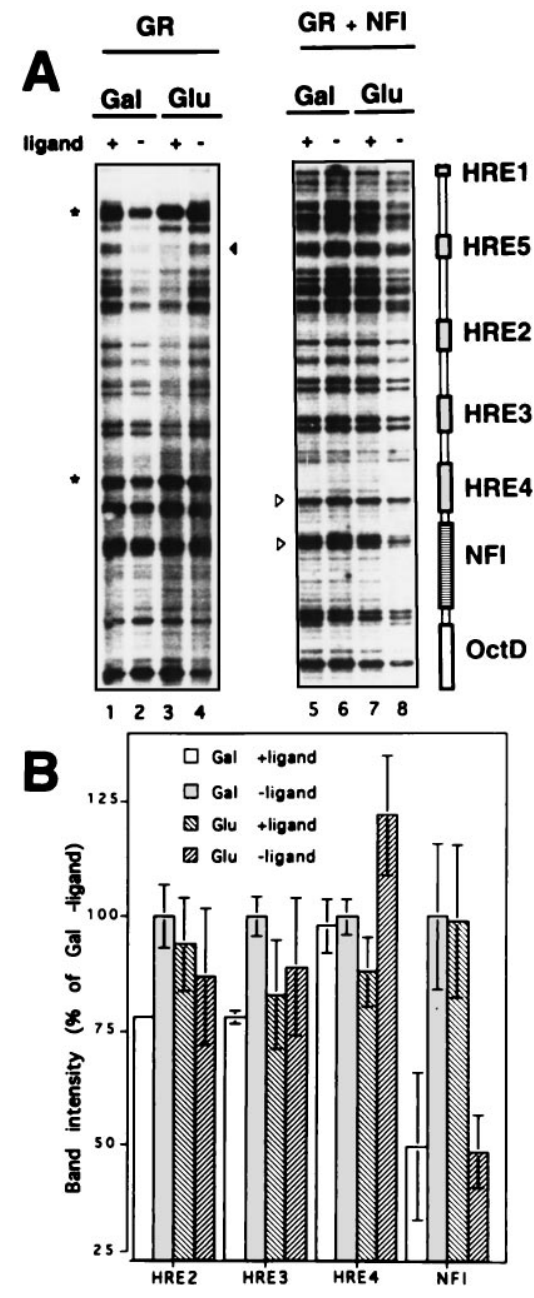

FIG. 4. Genomic footprinting over the MMTV promoter. (A Left) At low nucleosome density (glucose; Glu) there is a better access of GR to the HRE5 (marked by a triangle on the right) in response to ligand. The asterisks on the left mark two bands detected under all conditions, which do not correspond to guanines in the DNA sequence. Their identity is unknown. (Right) In the presence of NFI, at low nucleosome density, a cluster of guanines within the NFI site (triangle) is more protected before hormone treatment (lane 8) than after hormone induction (lane 7). The relevant guanine on HRE4 is marked by a triangle. $(B)$ PhosphorImager quantitation of the protection over the NFI site and the HRE2, -3 , and -4 , derived from three experiments similar to that shown in $A$ Right. The values were normalized for variations in loading using the strong band within the octamer distal site (OctD) at the bottom of the gel. The mean and standard deviation are shown. gene did not change the activity of the MMTV promoter (Fig. $3 B$ ). Thus, in the absence of NFI, GR binding and transactivation on the MMTV promoter do not require normal nucleosome density, but on the contrary are enhanced on promoters partially depleted of nucleosomes.

Nucleosome Depletion Compromises the Synergism Between GR and NFI Needed for Strong Induction. In yeast strains with physiological histone levels, the absence of NFI or a mutation of the NFI binding site reduces hormone induction of the MMTV promoter by $80 \%$, demonstrating that GR action involves a synergism with NFI (17). Having shown that the individual actions of NFI and GR are improved at low nucleosome density, we next asked whether the synergism between the two factors was similarly affected. Unexpectedly, nucleosome depletion resulted in suboptimal induction of transcription from the MMTV promoter in the presence of NFI, whereas glucose per se had no effect in yeast strains with a wild-type histone $\mathrm{H} 4$ gene (Fig. $3 D$ ). In the yeast strain used for these experiments the functional synergism between GR and NFI was 4- to 5-fold at normal nucleosome density and was completely abolished by nucleosome depletion (Fig. $3 C$ ). The residual induction seen in glucose medium is very similar to that observed in the absence of NFI (compare Fig. 3 B Left with $D$ Left) and likely reflects in part the improved GR function at low nucleosome density (see Fig. $3 A$ and $B$ ).

In genomic footprinting experiments, occupancy of the NFI site was enhanced after nucleosome depletion in the absence of hormone (Fig. 4A, compare lanes 6 and 8). Quantitation of

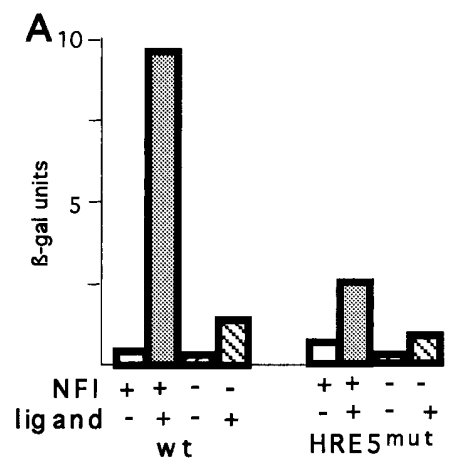

B
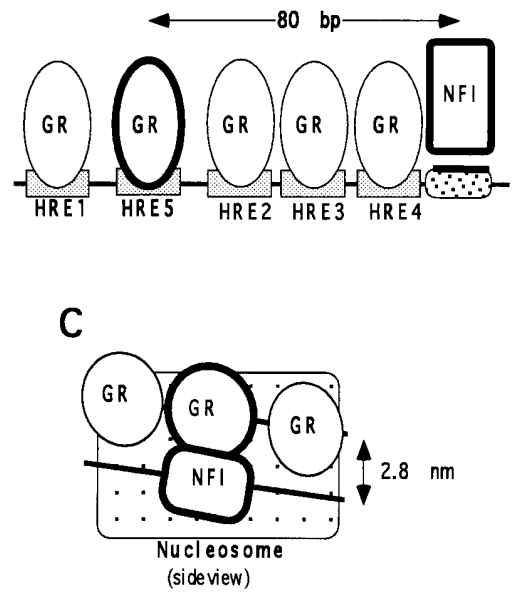

FIG. 5. HRE5 participates in synergistic induction by ligand activated GR. $(A)$ Influence of mutations in the HRE5 site on the activity of the MMTV promoter. The mutation is described in Materials and Methods. (B) Schematic array of sites: distance between HRE5 and the NFI site. $(C)$ Hypothetical model of GR and NFI bound to their cognate sites on the surface of a nucleosome. A GR (bold) bound to HRE5 would be close to the DNA-bound NFI. The model and the positions of the cis elements are not drawn to scale. 
the gel yields $51 \%$ protection following nucleosome depletion (Fig. $4 B$ ). At normal nucleosome density protection over the NFI site after hormone induction is only $50 \%$ (Fig. $4 B$ ) because the response of the yeast population is not homogeneous (17). Nevertheless, following hormone treatment, protection over the NFI site was reduced by nucleosome depletion to insignificant values (Fig. 4B), suggesting a competition between receptor and NFI at low nucleosome density (Fig. $4 A$ lanes 5 and 7). Due to known difficulties in footprinting DNA bound GR in vivo (16), the effects of ligand and of nucleosome depletion on the occupancy of the four classical HREs were less significant and did not allow safe conclusions.

A New HRE Participates in Induction by Ligand Activated GR. In the original experiments on GR binding to the MMTV promoter using DMS footprinting in vitro, a site was detected between position -156 and -144 , which when mutated did not show a phenotype in transient transfection assays $(30,33)$. Therefore, this site was considered a cryptic HRE. However, in genomic footprinting experiments in mammalian cells (16) and in yeast cells (17), changes in DMS sensitivity were found in the guanines over this sequence upon hormone induction, suggesting that hormone dependent interactions were taking place on this site (labeled HRE5 in Fig. 4A). Therefore, we tested the influence of mutations within this site on the induction of the MMTV reporter in yeast. In agreement with the footprint data, we found that mutations which interfere with GR binding to HRE5 (data not sown) have a significant effect on promoter activity, which is reduced to $25 \%$ of the activity of the wild-type promoter (Fig. $5 A)$. The effect of the HRE5 mutation is much less pronounced in the absence of NFI (Fig. 5A).

\section{DISCUSSION}

NFI binds with high affinity to the MMTV promoter DNA and transactivates efficiently under cell-free conditions $(13,14)$. However, like in nucleosome depleted yeast cells, neither DNA binding cooperativity nor synergism between GR and NFI have been detected on naked MMTV DNA. NFI binding to the promoter is rather hindered by bound GR (13). In intact cells with chromosomal copies of MMTV, the promoter is positioned in a nucleosome and free of factors in the absence of hormones $(15,16,34)$, though there is abundant NFI in the cell nucleus (35). Hormone induction leads to simultaneous occupancy of the HREs, and the NFI site, on the surface of a positioned nucleosome (16). These observations suggested that the organization of the MMTV promoter in chromatin not only represses the promoter prior to induction but could also play a positive role in hormonal activation (16). The results reported here support this hypothesis. The low activity of the MMTV promoter in the absence of hormone probably reflects the inability of NFI to access its cognate site when this is included in a positioned nucleosome (19-21, 36), because repression is partly relieved by nucleosome depletion. Derepression by nucleosome depletion has been reported for other inducible yeast promoters exhibiting nucleosome positioning, such as the PHO5 promoter, though in this case the main contribution may be an enhanced accessibility of the TATA box region (37). In the MMTV promoter this cannot be the only explanantion, because deletion of the HREs, leaving the NFI site and the TATA box intact, eliminates most of the nucleosome depletion effect. Prior to hormone induction, occupancy of the NFI site is enhanced upon nucleosome depletion, indicating that occlusion of this site by nucleosomes participates in constitutive repression of the promoter.

Hormone induction of a yeast promoter linked to HREs is improved by lowering the nucleosome density. The same is true for the MMTV promoter in the absence of NFI, suggesting that nucleosomes do actually hinder the NFI-independent activation of the promoter by liganded receptor. This effect could be partly due to a better binding of the receptor to the nucleosome- depleted promoter. It is known that GR binds to in vitro reconstituted MMTV nucleosomes with 3- to 8-fold lower affinity than to the same sequences as free DNA $(19,20,38)$. However, a more efficient interaction of the DNA-bound receptor with the general transcription factors could also contribute to the better inducibility observed at low nucleosomal density.

In marked contrast to the separate functions of NFI and GR, their cooperation on the MMTV promoter is not enhanced but rather abrogated by nucleosome depletion. The inhibition of the synergism between the two transcription factors under conditions of nucleosome depletion suggests that the functional interaction between GR and NFI may depend on the organization of the MMTV promoter sequence on the surface of a rearranged nucleosome. The elimination of synergism between the two factors was complete although the average nucleosome depletion was $<50 \%$. Two possible mechanisms could contribute to this strong effect. The MMTV promoter could be particularly sensitive to the inhibition of histone $\mathrm{H} 4$ synthesis and become depleted of nucleosomes to a much higher extent than the average DNA. There are no indications for this nucleosome being unusually unstable in vitro $(19,20)$, but the in vivo stability of this nucleosome is unknown. Alternatively, under conditions of partial nucleosome depletion there could be a competition between GR and NFI for binding to the MMTV promoter. Given that NFI is a weak transactivator in the absence of GR but binds the promoter DNA with high affinity, a competition could lead to an inhibition of the NFI-independent activation by liganded GR, and thus reduce the final level of activity. The genomic footprinting results support this notion. In the absence of GR, nucleosome depletion leads to higher occupancy of their NFI site. In contrast, depriving the MMTV promoter of nucleosomes reduces the occupancy of the NFI site in the presence of GR and ligand. Thus, nucleosome depletion reproduces within the cell nucleus the situation previously described on naked MMTV DNA in vitro.

There are few well defined examples for a positive role of nucleosomes in transcription. Deletion of the N-terminal tail of histone $\mathrm{H} 4$ reduces transactivation of the GAL1 promoter significantly, but this effect has been related to a change in nucleosome positioning that influences access to the TATA box (39). One interesting model may be the enhancer of the rat albumin gene, which in liver cells contains a positioned nucleosome, on which surface the relevant transcription factors can interact with their cognate DNA sequences (40). There is, however, little evidence for an active function of the nucleosome in this context.

How could the organization of the MMTV promoter sequence on the surface of a nucleosome facilitate transcription factor binding and functional synergism? A simple cooperativity between factors binding to adjacent sites $(41,42)$ is not sufficient to account for the synergism between GR and NFI, as it cannot be reproduced in the test tube with isolated MMTV nucleosomes and purified GR and NFI (20). It is likely that the observed synergism involves additional activities. A possible candidate is the ubiquitous SWI/SNF complex (43), which has been shown to facilitate GR induction in yeast and in animal cells $(44,45)$. However, no evidence for targeting of the SWI/SNF complex to the MMTV promoter has been produced, and other nucleosome remodeling activities have been recently identified which, in principle, could also participate in MMTV induction (46).

Once the nucleosome has been remodeled, there are two simple ways in which its organization could facilitate interactions between GR and NFI. It is possible that the curvature of DNA on the surface of the histone octamer may contribute to the synergism between receptors and NFI. Hormone receptors bound to HREs induce DNA bending toward the protein (47-49), and this bending may interfere with binding of NFI to an adjacent site. The wrapping of the double helix around the histone octamer bends the DNA in the opposite direction. 
This could not only account for the reduced affinity of hormone receptors for HREs organized in nucleosomes (38, 50 ), but could also prevent steric hindrance between GR and NFI by counteracting the receptor-induced DNA bending.

The nucleosome structure could also facilitate the functional interaction between GR and NFI simply by the close proximity of the two DNA superhelical turns on the surface of the nucleosome. This topology should bring distant HREs close to the NFI site. In this respect it is interesting that the cryptic HRE located around position -150 , now named HRE5, would come in close proximity to the NFI site on the surface of a nucleosome (Fig. 5C). Contrary to the other four HREs, HRE5 is not functional in transient transfection experiments with cultured cells (ref. 32 and unpublished results), probably reflecting the fact that in such experiments the promoter is not properly organized in chromatin (51). However, HRE5 participates in the synergistic activation by GR in yeast chromatin as demonstrated by the strong negative effect of mutations which abolish GR binding to this site. We also find that hormone induction leads to partial occupancy of the HRE5 both in yeast cells and animal cells (refs. 16 and 17 and unpublished results). Therefore, we have identified a hormone receptor binding site that seems to be fully functional only in the context of a nucleosomally organized MMTV promoter.

Apart from these simple mechanisms, it is also possible that structural components of chromatin could be directly involved in the interaction with sequence-specific transcription factors. In yeast, the N-terminal tails of histones $\mathrm{H} 3$ and $\mathrm{H} 4$ are required for activation of certain genes (32) and they have been shown to directly interact with other factors, for instance in the process of telomere silencing (7). Moreover, an interaction of NFI with the globular domain of histone $\mathrm{H} 3$ has been reported (9). If the hormone induced nucleosome rearrangement would involve the dissociation of a histone $2 \mathrm{~A}$ /histone $2 \mathrm{~B}$ dimer, it could expose a previously masked surface of histone $\mathrm{H} 3$ for an interaction with NFI. This would provide an attractive mechanism for an active participation of nucleosome components in the functional synergism between GR ad NFI. However, this explanation seems unlikely as the amino acid residues of NFI involved in interaction with histone $\mathrm{H} 3$ are located in the very C-terminal region of CTF1 (9), which is missing in the variant of NFI used for our experiments, namely CTF2 (9). While CTF2 interacts very weakly if at all with histone H3 (9), in terms of synergism with GR on the MMTV promoter, the two forms of NFI, CTF-1 and CTF-2, are indistinguishable (17). Of course, we cannot exclude that other components of the nucleosome do interact directly with NFI. The availability of yeast strains with mutations in the core histones and in other factors involved in chromatin dynamics should allow a genetic approach to these questions.

We thank Martin Funk (Institut für Molekularbiologie und Tumorforschung) and Michael Grunstein (University of California, Los Angeles) for yeast strains, constructs, and advice; Mathias Truss (Institut für Molekularbiologie und Tumorforschung) for constructing the HRE5 mutant; Andres Aguilera (Seville, Spain) for his generous support; and Jörg Klug, Reinhard Lührmann, and Rolf Müller (Institut für Molekularbiologie und Tumorforschung) for suggestions on the manuscript. S.C. was a postdoctoral fellow from the Human Capital and Mobility program of the European Union. This work was supported by grants from the Deutsche Forschungsgemeinschaft, the European Union, and the Fonds der Chemischen Industrie.

1. Van Holde, K. E. (1993) Nature (London) 362, 111-112.

2. Wolffe, A. P., Almouzni, G., Ura, K., Pruss, D. \& Hayes, J. J. (1993) Cold Spring Harbor Symp. Quant. Biol. 58, 225-235.

3. Thomas, G. H. \& Elgin, S. C. R. (1988) EMBO J. 7, 2191-2201.

4. Schild, C., Claret, F. X., Wahli, W. \& Wolffe, A. P. (1993) EMBO J. 12, 423-433.

5. Lu, Q., Wallrath, L. L. \& Elgin, S. C. R. (1995) EMBO J. 14, $4738-4746$
6. Kingston, R. E., Bunker, C. A. \& Imbalzano, A. N. (1996) Genes Dev. 10, 905-920.

7. Hecht, A., Laroche, T., Strahl-Bolsinger, S., Gasser, S. M. \& Grunstein, M. (1995) Cell 80, 583-592.

8. Edmondson, D. G., Smith, M. M. \& Roth, S. Y. (1996) Genes Dev. 10, 1247-1259.

9. Alevizopoulos, A., Dusserre, Y., Tsai-Pflugfelder, M., von der Weid, T., Wahli, W. \& Mermod, N. (1995) Genes Dev. 9, 3051-3066.

10. Beato, M., Herrlich, P. \& Schütz, G. (1995) Cell 83, 851-857.

11. Brüggemeier, U., Kalff, M., Franke, S., Scheidereit, C. \& Beato, M. (1991) Cell 64, 565-572.

12. Miksicek, R., Borgmeyer, U. \& Nowock, J. (1987) EMBO J. 6, 1355-1360.

13. Brüggemeier, U., Rogge, L., Winnacker, E. L. \& Beato, M. (1990) EMBO J. 9, 2233-2239.

14. Kalff, M., Gross, B. \& Beato, M. (1990) Nature (London) 344, 360-362.

15. Richard-Foy, H. \& Hager, G. L. (1987) EMBO J. 6, 2321-2328.

16. Truss, M., Bartsch, J., Schelbert, A., Haché, R. J. G. \& Beato, M. (1995) EMBO J. 14, 1737-1751.

17. Chávez, S., Candau, R., Truss, M. \& Beato, M. (1995) Mol. Cell. Biol. 15, 6987-6998.

18. Fragoso, G., John, S., Roberts, M. S. \& Hager, G. L. (1995) Genes Dev. 9, 1933-1947.

19. Perlmann, T. \& Wrange, Ö. (1988) EMBO J. 7, 3073-3079.

20. Piña, B., Brüggemeier, U. \& Beato, M. (1990) Cell 60, 719-731.

21. Archer, T. K., Cordingley, M. G., Wolford, R. G. \& Hager, G. L. (1991) Mol. Cell. Biol. 11, 688-698.

22. Roberts, M. S., Fragoso, G. \& Hager, G. L. (1995) Biochemistry 34, 12470-12480.

23. Candau, R., Chávez, S. \& Beato, M. (1996) J. Steroid Biochem. Mol. Biol. 57, 19-31.

24. Han, M. \& Grunstein, M. (1988) Cell 55, 1137-1145.

25. Kayne, P. S., Kim, U. J., Han, M., Mullen, J. R., Yoshizaki, F. \& Grunstein, M. (1988) Cell 55, 27-39.

26. Kim, U. J., Han, M., Kayne, P. \& Grunstein, M. (1988) EMBO J. 7, 2211-2219.

27. Schena, M. \& Yamamoto, K. R. (1988) Science 241, 965-967.

28. Altmann, H., Wendler, W. \& Winnacker, E. L. (1994) Proc. Natl. Acad. Sci. USA 91, 3901-3905.

29. Mumberg, D., Müller, R. \& Funk, M. (1994) Nucleic Acids Res. 22, 5767-5768.

30. Scheidereit, C. \& Beato, M. (1984) Proc. Natl. Acad. Sci. USA 81, 3029-3033.

31. Guarente, L. (1983) Methods Enzymol. 101, 81-191.

32. Durrin, L. K., Mann, R. K., Kayne, P. S. \& Grunstein, M. (1991) Cell 65, 1023-1031.

33. Chalepakis, G., Arnemann, J., Slater, E. P., Brüller, H., Gross, B. \& Beato, M. (1988) Cell 53, 371-382.

34. Cordingley, M. G., Riegel, A. T. \& Hager, G. L. (1987) Cell 48, 261-270.

35. Cordingley, M. G. \& Hager, G. L. (1988) Nucleic Acids Res. 16, 609-630.

36. Blomquist, P., Li, Q. \& Wrange, Ö. (1996) J. Biol. Chem. 271, 153-159.

37. Han, M., Kim, U. J., Kayne, P. \& Grunstein, M. (1988) EMBO J. 7, 2221-2228.

38. Perlmann, T. (1992) Proc. Natl. Acad. Sci. USA 89, 3884-3888.

39. Fisher-Adams, G. \& Grunstein, M. (1995) EMBO J. 14, 1468-1477.

40. McPherson, C. E., Shim, E. Y., Friedman, D. S. \& Zaret, K. S. (1993) Cell 75, 387-398.

41. Adams, C. C. \& Workman, J. L. (1995) Mol. Cell. Biol. 15,1405-1421.

42. Polach, K. J. \& Widom, J. (1995) J. Mol. Biol. 254, 130-149.

43. Winston, F. \& Carlson, M. (1992) Trends Genet. 8, 387-391.

44. Yoshinaga, S. K., Peterson, C. L., Herskowitz, I. \& Yamamoto, K. R. (1992) Science 258, 1598-1604.

45. Muchardt, C. \& Yaniv, M. (1993) EMBO J. 12, 4279-4290.

46. Tsukiyama, T. \& Wu, C. (1995) Cell 83, 1011-1020.

47. Piña, B., Barettino, D., Truss, M. \& Beato, M. (1990) J. Mol. Biol. 216, 975-990.

48. Nardulli, A. M. \& Shapiro, D. J. (1992) Mol. Cell. Biol. 12, 2037-2042.

49. Prendergast, P., Pan, Z. X. \& Edwards, D. P. (1996) Mol. Endocrinol. 10, 393-407.

50. Li, Q. \& Wrange, Ö. (1995) Mol. Cell. Biol. 15, 4375-4384.

51. Archer, T. K., Lefebvre, P., Wolford, R. G. \& Hager, G. L. (1992) Science 255, 1573-1576. 